\title{
BMJ Open Risk factors for delirium among older adults in the emergency department: a systematic review protocol
}

\author{
Lucas Oliveira J e Silva (D) , ${ }^{1}$ Michelle J Berning, ${ }^{1,2}$ Jessica A Stanich, ${ }^{1}$ \\ Danielle J Gerberi (D) , 3 Jin Han, ${ }^{4,5}$ Fernanda Bellolio ${ }^{1,6}$
}

To cite: Oliveira J e Silva L, Berning MJ, Stanich JA, et al. Risk factors for delirium among older adults in the emergency department: a systematic review protocol. BMJ Open 2020;10:e039175. doi:10.1136/ bmjopen-2020-039175

- Prepublication history and additional material for this paper are available online. To view these files, please visit the journal online (http://dx.doi. org/10.1136/bmjopen-2020039175).

Received 07 April 2020

Revised 29 May 2020

Accepted 10 June 2020

Check for updates

(c) Author(s) (or their employer(s)) 2020. Re-use permitted under CC BY-NC. No commercial re-use. See rights and permissions. Published by BMJ.

${ }^{1}$ Department of Emergency Medicine, Mayo Clinic, Rochester, Minnesota, USA ${ }^{2}$ Medical School, University of Minnesota, Minneapolis,

Minnesota, USA

${ }^{3}$ Mayo Clinic Libraries, Mayo Clinic, Rochester, Minnesota USA

${ }^{4}$ Department of Emergency Medicine, Vanderbilt University, Nashville, Tennessee, USA

${ }^{5}$ Geriatric Research, Education, and Clinical Center, VA Tennessee Valley Healthcare System Nashville Campus, Nashville, Tennessee, USA

${ }^{6}$ Department of Health Sciences Research, Mayo Clinic, Rochester, Minnesota, USA

Correspondence to

Dr Lucas Oliveira J e Silva;

lojesilva@gmail.com

\section{ABSTRACT}

Introduction Delirium is commonly missed in older adults presenting to the emergency department (ED). Although current recommendations for active screening of delirium in the ED, this might not be feasible or practical. Identifying patients at high risk for prevalent and incident delirium in the ED will help to improve the screening process and to build interventions. There is currently scattered synthesis of evidence on risk factors associated with delirium in the ED. To address this gap, we are conducting a systematic review to describe the risk factors (patient vulnerability factors and precipitating factors) for delirium in the ED. Methods and analysis A literature search was performed from inception to March 2020 in Ovid EBM Reviews, Ovid EMBASE, Ovid MEDLINE, Scopus and Web of Science. We will include original research studies that report a quantitative relationship between at least one risk factor and delirium in the ED setting. Two investigators will use eligibility criteria from this protocol to independently screen titles and abstracts, and select studies based on full-text review of potentially eligible studies. After arriving at a final set of included studies, two investigators will extract data using a standardised data collection form. If appropriate, data regarding each risk factor will be pooled through a random-effect meta-analysis. The Grading of Recommendations Assessment, Development and Evaluation approach will be used to evaluate the overall quality of evidence.

Ethics and dissemination To our knowledge, this will be the first systematic review evaluating risk factors for prevalent and incident delirium specifically related to the ED setting. Results of this study will aid in the identification of older adults at risk for delirium in the ED. We aim to publish the results of this systematic review in a peer-reviewed journal with good visibility for the fields of emergency medicine and geriatrics.

PROSPERO registration number

CDR42020175261

\section{INTRODUCTION}

\section{Rationale}

Delirium is an acute condition characterised by rapid change in brain function, which primarily affects the ability to focus attention. Its clinical course involves fluctuating symptoms over the course of a day with periods of lucidity followed by increased confusion.

\section{Strengths and limitations of this study}

- While risk factors for prevalent and incident delirium may overlap, this study will help to identify emergency department (ED)-related iatrogenic risk factors for incident delirium, which may be useful to develop future interventions.

- This study will inform researchers for the future development of clinical prediction rules when identifying older adults at risk for delirium in the ED.

- This review will be limited to the ED setting.

- Primary studies may not have a well-defined differentiation between incident and prevalent delirium in the ED, which may limit the interpretation of the findings of this systematic review.

- Given the potential heterogeneity across studies, we may not be able to perform meta-analysis.

Delirium is a major concern for older adults presenting to emergency departments (ED) across the USA, with estimates that up to $10 \%$ of older adults present to the ED with this condition. ${ }^{1-4}$ With older age, particularly those over 75 years, there is a higher risk of developing delirium in the hospital. ${ }^{5}$ The diagnosis of delirium in the ED is associated with increased mortality, prolonged hospitalisation, decreased functionality, cognitive impairment and increased healthcare costs. ${ }^{6-9}$

Currently, there is a gap in recognising and addressing delirium in the ED. ${ }^{1-4}$ Clinical presentation of delirium can be subtle, particularly when the patient is unknown to the provider and there are no family members or caregivers to provide history, which is often the case in the emergency setting. Also, up to $90 \%$ of patients present with hypoactive psychomotor subtype, so instead of agitation these patients will be quiet and withdrawn. ${ }^{10}$ Active screening for this condition is then needed in the emergency setting as recommended by the Geriatric Emergency Department Guidelines. ${ }^{11}$

Screening for every single older adult in the ED might not be feasible or effective. 
However, performing delirium assessment on a subset of high-risk older patients may be practical and beneficial. Most studies evaluating risk factors for delirium have been conducted in other settings like the intensive care unit (ICU) or hospital wards.

A systematic review performed by Ahmed et al evaluated risk factors for incident delirium among older adults admitted to the hospital. In their pooled analyses, they found that dementia, illness severity, visual impairment, urinary catheterisation, low albumin level and length of hospital stay were significantly associated with incident delirium in the inpatient setting. ${ }^{5}$ Two reviews have focused on identifying risk factors among critically ill patients admitted to the ICU. ${ }^{12}{ }^{13}$ Mattar et al found 22 studies across three different ICU settings: medical ICU, surgical ICU and cardiac ICU. A meta-analysis was not performed but they found that the use of benzodiazepines and opioids were important risk factors for delirium across all these settings. ${ }^{12}$ Zaal $e t$ al evaluated risk factors for delirium in the ICU setting as a whole. They included a total of 33 studies, and found strong evidence that age, dementia, hypertension, pre-ICU emergency surgery or trauma, illness severity, mechanical ventilation, metabolic acidosis, delirium on the prior day and coma are risk factors for delirium in the ICU. The authors did not perform a meta-analysis given the high heterogeneity across studies. ${ }^{13}$ Krewulak et al comprehensively evaluated the risk factors and outcomes among delirium subtypes in adult ICUs. They included a total of 20 studies, and found inconsistent and heterogeneous results, pointing out for the need to standardise reporting and methodology in studies looking at different subtypes of delirium. ${ }^{14}$ Delirium risk factors have also been evaluated in specific surgical population such as vascular surgery. ${ }^{15}$

None of the previous systematic reviews have focused or included studies evaluating ED geriatric patients. Only narrative reviews have evaluated risk factors for delirium in the ED, but they failed to include the whole body of evidence. ${ }^{16} 17$ Also, they have not taken into consideration the differences in risk factors for prevalent and incident delirium. Prevalent delirium is defined as delirium on patient presentation in the ED. Incident delirium is defined as those patients who were initially non-delirious and developed delirium during the ED clinical course or during the hospitalisation. While risk factors for prevalent and incident delirium may overlap, incident delirium will also include ED-related iatrogenic risk factors (figure 1). Identifying such risk factors may be useful to develop future ED-based interventions.

Although risk factors for delirium might be similar across settings, there is a need to understand if there are important differences in the ED. The ED has a very unique environment when compared with inpatient floors or ICUs. Hospital-based studies often enrol patients up to 48 hours after admission. ${ }^{18-21}$ Given its fluctuant nature, the delirium status of a patient after 48 hours of admission might be completely different from the delirium status on arrival in the ED or throughout the ED length of
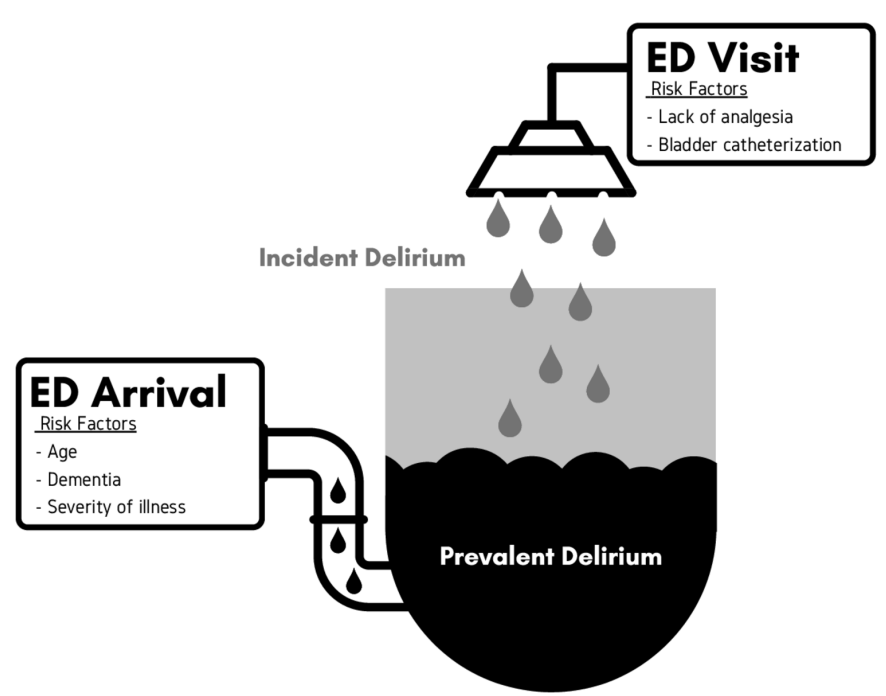

Figure 1 Conceptual model of risk factors and prevalent and incident delirium from the ED perspective. ED, emergency department.

stay, which mainly explains the limited generalisability of these studies to the ED patient. Besides that, healthcare providers in the ED may have limited information about the patient (eg, if the patient cannot provide a reliable history and/or no family members are present). Therefore, risk factors identified in other settings may be less relevant or not applicable in the ED context.

This review will inform researchers and clinicians about who are the high-risk geriatric patients for delirium in the ED setting. This will aid the future development of risk stratification tools that might be used to decide who needs to be actively screened for delirium in the ED. Despite guidelines' recommendations to screen every single older adult for delirium, ${ }^{11}$ there is a need to implement an effective delirium screening strategy in the ED.

\section{Review question}

What are the risk factors for prevalent and incident delirium among older adults in the ED setting?

\section{METHODS}

\section{Standards}

The methodology of this review is based on guidance of the Cochrane handbook, ${ }^{22}$ the Joanna Briggs Institute (JBI) Reviewer's Manual on systematic reviews of aetiology and risk, ${ }^{23}{ }^{24}$ and the Conducting Systematic Reviews and Meta-Analyses of Observational Studies of Aetiology. ${ }^{25}$ This protocol manuscript follows the Preferred Reporting Items for Systematic Reviews and Meta-Analyses Protocols (PRISMA) guidelines. ${ }^{26}$ The final publication of the review will adhere to the PRISMA statement. ${ }^{27}$

\section{Eligibility criteria}

We will include studies that evaluated risk factors for either prevalent or incident delirium in the ED setting among older adults (age $\geq 65$ ). Prevalent delirium will be defined as delirium present on ED arrival. This will 
include studies where delirium screening was performed on arrival in the ED using standardised delirium assessments. Incident delirium will be defined as delirium that occurs either during the ED length of stay or during the subsequent hospitalisation for those ED patients who are admitted to the hospital. If a clear timeline is not well established by the study on when delirium was assessed during the ED course, we will define it more broadly as delirium in the ED rather than incident vs prevalent. Only studies in which a standardised delirium diagnostic/assessment tool was used to reach a diagnosis of delirium will be considered. These tools include the following: Confusion Assessment Method (CAM),${ }^{28}$ CAM for the ICU (CAM-ICU), ${ }^{29}$ Delirium Triage Screen, ${ }^{30}$ brief CAM, ${ }^{30} 3$ min Diagnostic Interview for CAM (3D$\mathrm{CAM})^{31}$ and the $4 \mathrm{AT} \cdot{ }^{32}$ Studies of either delirium tremens or excited delirium will be excluded given the significant difference in its pathophysiology.

We will include original observational research (casecontrol, cohort and cross-sectional studies) that report a quantitative relationship between at least one risk factor and delirium in the ED setting. Interventional studies, if available, will be included, however, these are less likely to assess risk factors. Conference abstracts will be considered. Studies in all languages will be considered. There will be no restrictions for year of publication. Studies in the primary care, inpatient or ICU settings will be excluded. The following article types will be excluded: narrative or systematic reviews, reports from organisations, qualitative articles, editorials, commentaries, letters to the editor and case reports.

\section{Risk factors}

We will consider any risk factor under evaluation of the studies included. These will be divided by patient vulnerability factors and precipitating factors. Patient vulnerability factors include variables such as age and presence of comorbidities (eg, dementia, visual impairment, hearing impairment), while precipitating factors include variables such as current infectious process or interventions (eg, urinary catheters). The inter-relationship between these two broad categories of risk factors seem to play an important role in the development of delirium. ${ }^{33}$

\section{Search strategy}

The literature search will contain three parts: (1) the databases including EBM Reviews, EMBASE, MEDLINE, Scopus and Web of Science; (2) the reference lists of relevant papers; (3) the grey literature sources. The reference lists of relevant papers, especially other literature reviews on the topic, may identify citations which did not appear during a database search. Conference abstracts will be reviewed, including those within the searched databases and also, we will hand-search websites and national guidelines from the main Geriatrics and Emergency Medicine societies. There will be no time restriction for the search.

A preliminary literature search was developed and executed by an academic medical librarian (DJG) with input from the study investigators. Concepts of risk factors for delirium in older adults presenting to the ED were searched. The search strategies were created using a combination of keywords and standardised index terms. Searches were run in March 2020 in Ovid EBM Reviews, Ovid EMBASE (1974+), Ovid MEDLINE (1946+ including e-pub ahead of print, in-process \& other non-indexed citations), Scopus (1970+) and Web of Science (1975+). All results were exported to EndNote where duplicates were removed, yielding 4340 citations. Search strategies are provided in the online supplementary appendix.

\section{Study selection, data extraction and risk of bias}

For primary study selection, two reviewers (LOJeS and MJB) will consider the potential eligibility of each of the abstracts and titles that result from executing the search strategy (phase I). Reviewers will request the full-text versions of all potentially eligible studies. Disagreements during phase I will be retrieved in full text for evaluation. If an article meets inclusion criteria but it is not available through our institution library access, authors will be contacted by electronic mail to obtain the full text.

For secondary study selection, two reviewers (LOJeS and MJB) working independently and blindly will consider the full-text reports (all available versions of each study) for eligibility (phase II). The reviewers will calibrate their judgements using a smaller set of reports. Subsequently, disagreements will be harmonised by consensus and discussion with the senior author. Inter-rater agreement will be measured using Cohen's kappa coefficient for phase II. Standardised forms for both phase I and phase II will be developed.

After achieving the final number of studies to be included in our systematic review, data extraction will occur. A specific data extraction tool will be developed for the purpose of this review. Data collection form will be developed in Microsoft Excel 2016 software. This tool will be tested on a set of 2-4 articles prior to use, and the tool will then be modified as needed. Data extraction will be done by two investigators (LOJeS and MJB) independently. The following information will be extracted from each study: study design, sample size, inclusion/ exclusion criteria, any risk factor evaluated, outcome measure (ie, delirium diagnostic tool, presence/absence of delirium, whether or not it was possible to determine prevalent vs incident delirium, time frame for the ascertainment of delirium, overall frequency of delirium, raw numbers regarding risk factors in delirium vs nondelirium patients as well as results from univariate and multivariate analyses). Extraction of effect measures (both ratio measures and difference measures) will be done following Cochrane ${ }^{34}$ and $\mathrm{JBI}^{24}$ recommendations. Both unadjusted and adjusted estimates will be extracted. Confounding is a key item in the analysis of risk factors for delirium and we will collect the list of confounders that each study considered. Data regarding who performed the delirium assessment (ie, research assistant, registered nurse, etc) will also be obtained. If data are not available 
in the published manuscript, authors will be contacted by electronic mail to request the information needed.

As for the risk of bias assessment at the study level, a modified version of Newcastle-Ottawa Scale will be used for case-control, cohort and cross-sectional studies. ${ }^{35}$ Key domains will include assessments of sample selection, risk factors (exposures) ascertainment, comparability of cohorts on the basis of design or analysis and delirium (outcome) ascertainment. Each domain will be judged as low, unclear or high risk of bias. For comparability of cohorts on the basis of analysis, studies that do not adjust risk factors for potentially confounding variables will be deemed to have higher risk of bias. For example, increased age and dementia are well-known risk factors for delirium across all settings and studies evaluating other risk factors need to adjust for these variables. Although not likely to have interventional studies evaluating risk factors, if included the Cochrane tool will be used to evaluate risk of bias for this study design. ${ }^{36}$ Risk of bias assessments will be performed by two investigators (LOJeS and MJB) independently.

\section{Data synthesis}

After evaluating the availability of data and the heterogeneity from included studies, we will evaluate whether or not it is appropriate to conduct a meta-analysis to determine the relationship between risk factors and delirium. If the association measures have enough similarity, we will generate meta-analytic estimates (pooled ORs) for each risk factor using random-effects meta-analysis as described by DerSimonian and Laird. ${ }^{37}$ Enough similarity will be defined as studies looking at the same risk factors with similar study populations. Delirium may be measured by different standardised instruments and meta-analytical pooling will be considered only if the same or similar instrument was used. The random-effect model is a conservative choice given expected heterogeneity within and across studies. We will measure inconsistency and statistical heterogeneity by estimating the $\mathrm{I}^{2}$ index and Cochrane $Q$ test. ${ }^{38}$ We will use RevMan V.5.3 and/or MetaXL software to conduct the analyses. Metaanalytical estimates will be obtained separately for unadjusted and adjusted data. Comparisons of adjusted and crude estimates may allow insights into the importance of confounding. ${ }^{25}$

We anticipate significant methodological differences across studies, which might preclude our ability to perform meta-analysis of associations between risk factors and ED delirium. As an alternative to synthesise data regarding risk factors for ED delirium, we aim to use harvest plots. Harvest plot is a novel approach to graphically summarise the results of systematic reviews and it may be particularly useful for systematic reviews addressing a broader research question. ${ }^{23}$ Also, harvest plots can be used to synthesise results when only direction of the association is reported, or when there is inconsistency in the effect measures or data reported across studies. ${ }^{39}$
Subgroup analysis of interest include: prevalent versus incident delirium, patients with versus without dementia, study design (eg, retrospective vs prospective observational studies) and study quality (eg, low vs high risk of bias).

We will only analyse publication bias through funnel plots for those meta-analytic estimates based on more than 10 studies. A small number of studies make analysis of funnel plots unreliable. ${ }^{40}$

\section{Summarising evidence}

We will use the Grading of Recommendations, Assessment, Development and Evaluations (GRADE) approach for grading the quality of evidence taking into consideration other factors beyond the risk of bias assessment (ie, imprecision, inconsistency, indirectness and publication bias). Best evidence regarding prognostic or risk factors usually originates from observational studies, and for this systematic review such studies may start with high certainty ratings. ${ }^{41}$ Other domains such as magnitude of effect and effect of plausible residual confounding will also be considered. ${ }^{41}{ }^{42}$ For example, if the effect estimate is strong (ie, relative effect size over 2.0), we will consider upgrading the certainty level. Depending on issues related to these domains, certainty in the evidence will be ultimately designated as high, moderate, low or very low. ${ }^{41}$ The GRADEpro online software (https://community. cochrane.org/help/tools-andsoftware/gradepro-gdt) will be used to build evidence summaries. The quality of evidence (ie, certainty in estimates) will be evaluated for each association between risk factors and delirium in the ED setting. If the significant heterogeneity across studies preclude us from performing meta-analysis, we will follow the framework of Murad $e t$ al for rating the certainty in evidence in the absence of a single estimate of effect. ${ }^{43}$

\section{Current study status}

At the time of writing this manuscript, literature search was completed by the medical librarian and phase I (screening of titles and abstracts) was in progress.

\section{Ethics and dissemination}

This study involves only analysis of data from published studies and was deemed to be exempt from our institutional review board. Results of this systematic review will be published in a peer-reviewed journal with good visibility for the fields of emergency medicine and geriatrics.

\section{Patient and public involvement}

There was no patient or public involvement in the development of this protocol.

\section{DISCUSSION}

To our knowledge, this will be the first systematic review specifically evaluating risk factors for delirium in the ED setting. Results of this study will inform policy-makers, researchers and emergency physicians to evolve strategies to better identify older adults with delirium in the ED as 
well as implement interventions to reduce the burden of precipitating factors and, consequently, of delirium in the ED.

Clinical prediction instruments are most useful when there is evidence that clinicians are failing to accurately diagnose a condition, and when there are serious consequences associated with the missing diagnosis. ${ }^{44}$ Emergency physicians miss delirium in $57 \%-83 \%$ of cases during routine clinical care. ${ }^{1045} 46$ A systematic review identified 28 delirium prediction models in the literature, however, none of these models were built for the ED setting. ${ }^{47}$ The development of a clinical prediction instrument based on risk factors found on a comprehensive systematic review of the literature might help to identify delirium screening targets in the very early period of ED clinical course. The identification of risk factors for delirium in the ED will help providers to identify patients who are at high risk for prevalent and incident delirium in the $\mathrm{ED}$, and then require active screening. Also, this could facilitate the life of emergency physicians and optimise delirium screening in the ED. Early identification of these patients who are more likely to be delirious on arrival in the ED may allow for earlier intervention and thus potentially improve outcomes.

Possible limitations of this systematic review are several. First, some studies might not have a well-defined differentiation between incident and prevalent delirium in the ED. Second, the significant heterogeneity across studies may preclude us to perform meta-analyses. Lastly, the quality of included studies is expected to be low and we anticipate a low level of certainty about the associations of certain risk factors and ED delirium.

Twitter Lucas Oliveira J e Silva @lucasojesilva12 and Fernanda Bellolio @ mfbellolio

Contributors Conceptualisation: LOJeS and FB. Formal analysis: LOJeS and FB. Investigation: LOJeS, MJB, JAS, JH and FB. Literature search: DJG. Methodology: LOJeS and FB. Project administration: LOJeS. Supervision: FB. Validation: FB. Writing-original draft: LOJeS. Writing-review and editing: LOJeS, MJB, JAS, DJG, $\mathrm{JH}$ and FB. Guarantor: LOJeS and FB. All authors provided critical revision and contribution for important intellectual content.

Funding This study received funding through the Kern Society Innovation Award, from the Mayo Clinic Robert D. and Patricia E. Kern Center for the Science of Health Care Delivery. The funders' and authors' institutions are not responsible for its content.

Competing interests None declared.

Patient and public involvement Patients and/or the public were not involved in the design, or conduct, or reporting, or dissemination plans of this research.

Patient consent for publication Not required.

Provenance and peer review Not commissioned; externally peer reviewed.

Open access This is an open access article distributed in accordance with the Creative Commons Attribution Non Commercial (CC BY-NC 4.0) license, which permits others to distribute, remix, adapt, build upon this work noncommercially, and license their derivative works on different terms, provided the original work is properly cited, appropriate credit is given, any changes made indicated, and the use is non-commercial. See: http://creativecommons.org/ licenses/by-nc/4.0/.

\section{ORCID iDs}

Lucas Oliveira J e Silva http://orcid.org/0000-0001-5388-9163

Danielle J Gerberi http://orcid.org/0000-0002-1522-3915

\section{REFERENCES}

1 Barron EA, Holmes J. Delirium within the emergency care setting, occurrence and detection: a systematic review. Emerg Med $J$ 2013;30:263-8.

2 LaMantia MA, Messina FC, Hobgood CD, et al. Screening for delirium in the emergency department: a systematic review. Ann Emerg Med 2014;63:551-60.

3 Pérez-Ros P, Martínez-Arnau FM. Delirium assessment in older people in emergency departments. A literature review. Diseases 2019;7:14.

4 Mariz J, Costa Castanho T, Teixeira J, et al. Delirium diagnostic and screening instruments in the emergency department: an up-to-date systematic review. Geriatrics 2016;1:22.

5 Ahmed S, Leurent B, Sampson EL. Risk factors for incident delirium among older people in acute hospital medical units: a systematic review and meta-analysis. Age Ageing 2014;43:326-33.

$6 \mathrm{Han} \mathrm{JH}$, Shintani A, Eden S, et al. Delirium in the emergency department: an independent predictor of death within 6 months. Ann Emerg Med 2010;56:244-52.

7 Han JH, Eden S, Shintani A, et al. Delirium in older emergency department patients is an independent predictor of hospital length of stay. Acad Emerg Med 2011;18:451-7.

8 Vida S, Galbaud du Fort G, Kakuma R, et al. An 18-month prospective cohort study of functional outcome of delirium in elderly patients: activities of daily living. Int Psychogeriatr 2006;18:681-700.

9 Naughton BJ, Moran MB, Kadah H, et al. Delirium and other cognitive impairment in older adults in an emergency department. Ann Emerg Med 1995;25:751-5.

$10 \mathrm{Han}$ JH, Zimmerman EE, Cutler N, et al. Delirium in older emergency department patients: recognition, risk factors, and psychomotor subtypes. Acad Emerg Med 2009;16:193-200.

11 American College of Emergency Physicians, American Geriatrics Society, Emergency Nurses Association, et al. Geriatric emergency department guidelines. Ann Emerg Med 2014;63:e7-25.

12 Mattar I, Chan MF, Childs C. Risk factors for acute delirium in critically ill adult patients: a systematic review. ISRN Critical Care 2013:2013:1-10.

13 Zaal IJ, Devlin JW, Peelen LM, et al. A systematic review of risk factors for delirium in the ICU. Crit Care Med 2015;43:40-7.

14 Krewulak KD, Stelfox HT, Ely EW, et al. Risk factors and outcomes among delirium subtypes in adult ICUs: a systematic review. J Crit Care 2020;56:257-64.

15 Oldroyd C, Scholz AFM, Hinchliffe RJ, et al. A systematic review and meta-analysis of factors for delirium in vascular surgical patients. $J$ Vasc Surg 2017;66:1269-79.

16 Vasilevskis EE, Han JH, Hughes CG, et al. Epidemiology and risk factors for delirium across Hospital settings. Best Pract Res Clin Anaesthesiol 2012;26:277-87.

17 Rosen T, Connors S, Clark S, et al. Assessment and management of delirium in older adults in the emergency department: literature review to inform development of a novel clinical protocol. Adv Emerg Nurs J 2015;37:183-96.

18 Murray AM, Levkoff SE, Wetle TT, et al. Acute delirium and functional decline in the hospitalized elderly patient. $J$ Gerontol 1993;48:M181-6.

19 Inouye SK, Rushing JT, Foreman MD, et al. Does delirium contribute to poor hospital outcomes? A three-site epidemiologic study. J Gen Intern Med 1998;13:234-42.

20 Buurman BM, Hoogerduijn JG, de Haan RJ, et al. Geriatric conditions in acutely hospitalized older patients: prevalence and oneyear survival and functional decline. PLoS One 2011;6:e26951.

21 González M, Martínez G, Calderón J, et al. Impact of delirium on short-term mortality in elderly inpatients: a prospective cohort study. Psychosomatics 2009;50:234-8.

22 Higgins J, Green S. Cochrane Handbook for systematic reviews of interventions version 5.1.0. Cochrane Collab, 2011. Available: www. handbook.cochrane.org

23 Moola S, Munn Z, Sears K, et al. Conducting systematic reviews of association (etiology): the Joanna Briggs Institute's approach. Int $J$ Evid Based Healthc 2015;13:163-9.

24 Chapter 7: Systematic reviews of etiology and risk - JBI Reviewer's Manual - JBI GLOBAL WIKI. Available: https://wiki.joannabriggs.org/ display/MANUAL/Chapter+7\%3A+Systematic+reviews+of+etiology+ and+risk [Accessed 25 May 2020].

25 Dekkers OM, Vandenbroucke JP, Cevallos M, et al. COSMOS-E: guidance on conducting systematic reviews and meta-analyses of observational studies of etiology. PLoS Med 2019;16:e1002742.

26 Shamseer L, Moher D, Clarke M, et al. Preferred reporting items for systematic review and meta-analysis protocols (PRISMA-P) 2015: elaboration and explanation. BMJ2015;349:g7647. 
27 Moher D, Liberati A, Tetzlaff J, et al. Preferred reporting items for systematic reviews and meta-analyses: the PRISMA statement. BMJ 2009;339:b2535.

28 Inouye SK, van Dyck CH, Alessi CA, et al. Clarifying confusion: the confusion assessment method. A new method for detection of delirium. Ann Intern Med 1990;113:941-8.

29 Ely EW, Margolin R, Francis J, et al. Evaluation of delirium in critically ill patients: validation of the confusion assessment method for the intensive care unit (CAM-ICU). Crit Care Med 2001;29:1370-9.

$30 \mathrm{Han} \mathrm{JH}$, Wilson A, Vasilevskis EE, et al. Diagnosing delirium in older emergency department patients: validity and reliability of the delirium triage screen and the brief confusion assessment method. Ann Emerg Med 2013;62:457-65.

31 Marcantonio ER, Ngo LH, O'Connor M et al. 3D-CAM: derivation and validation of a 3-minute diagnostic interview for CAM-defined delirium: a cross-sectional diagnostic test study. Ann Intern Med 2014;161:554-61.

32 Bellelli G, Morandi A, Davis DHJ, et al. Validation of the 4AT, a new instrument for rapid delirium screening: a study in 234 hospitalised older people. Age Ageing 2014;43:496-502.

33 Inouye SK. Precipitating factors for delirium in hospitalized elderly persons. JAMA 1996;275:852

34 Higgins J, Li T, Deeks J. Chapter 6: Choosing effect measures and computing estimates of effect. In: Higgins JPT, Thomas J, Chandler J, et al, eds. Cochrane Handbook for systematic reviews of interventions version 6.0, 2019.

35 Stang A. Critical evaluation of the Newcastle-Ottawa scale for the assessment of the quality of nonrandomized studies in metaanalyses. Eur J Epidemiol 2010;25:603-5.

36 Sterne JAC, Savović J, Page MJ, et al. Rob 2: a revised tool for assessing risk of bias in randomised trials. BMJ 2019;366:14898.
37 DerSimonian R, Laird N. Meta-analysis in clinical trials revisited. Contemp Clin Trials 2015:45:139-45.

38 Higgins JPT, Thompson SG, Deeks JJ, et al. Measuring inconsistency in meta-analyses. BMJ 2003;327:557-60.

39 McKenzie J, Brennan S. Chapter 12: Synthesizing and presenting findings using other methods. In: Higgins JPT, Thomas J, Chandler J, et al, eds. Cochrane Handbook for systematic reviews of interventions version 6.0, 2019. www.training.cochrane.org/ handbook

40 Lau J, loannidis JPA, Terrin N, et al. The case of the misleading funnel plot. BMJ 2006;333:597-600.

41 Foroutan F, Guyatt G, Zuk V, et al. Grade guidelines 28: use of grade for the assessment of evidence about prognostic factors: rating certainty in identification of groups of patients with different absolute risks. J Clin Epidemiol 2020;121:62-70.

42 Balshem H, Helfand M, Schünemann HJ, et al. Grade guidelines: 3. rating the quality of evidence. J Clin Epidemiol 2011;64:401-6.

43 Murad MH, Mustafa RA, Schünemann HJ, et al. Rating the certainty in evidence in the absence of a single estimate of effect. Evid Based Med 2017;22:85-7.

44 Cowley LE, Farewell DM, Maguire S, et al. Methodological standards for the development and evaluation of clinical prediction rules: a review of the literature. Diagn Progn Res 2019;3:1-23.

45 Kakuma R, du Fort GG, Arsenault L, et al. Delirium in older emergency department patients discharged home: effect on survival. J Am Geriatr Soc 2003;51:443-50.

46 Hustey FM, Meldon SW, Smith MD, et al. The effect of mental status screening on the care of elderly emergency department patients. Ann Emerg Med 2003;41:678-84.

47 Lindroth H, Bratzke L, Purvis S, et al. Systematic review of prediction models for delirium in the older adult inpatient. BMJ Open 2018;8:e019223. 